\title{
Metodología bayesiana para la optimización simultánea de múltiples respuestas
}

\author{
Jorge Limón ${ }^{(1)}$, Manuel A. Rodriguez ${ }^{(2)}$, Jaime Sánchez ${ }^{(2)}$, Diego A. Tlapa ${ }^{(1)}$ \\ (1) Universidad Autónoma de Baja California, Facultad de Ingeniería, Arquitectura y Diseño \\ Ensenada, Km. 103 Carretera Tijuana-Ensenada, Baja California-México. \\ (e-mail jorge.limon@uabc.edu.mx) \\ (2) Instituto Tecnológico de Ciudad Juárez, Blvd. Tecnológico No. 1340, Ciudad Juárez, \\ Chihuahua-México.
}

Recibido Jul. 12, 2011; Aceptado Ago. 22, 2011; Versión Final recibida Sep. 16, 2011

\section{Resumen}

Se muestra la aplicación de una metodología bayesiana para la optimización simultánea de múltiples respuestas. Se modifica una propuesta anterior, introduciendo una modificación que la hace más flexible, ya que permite trabajar con el caso en que no todas las variables consideradas en el estudio son igualmente importantes. Para incorporar estas diferencias en cuanto a la relevancia de las variables de respuesta analizadas se utiliza la media geométrica ponderada de las probabilidades de que cada variable cumpla con su especificación. Con esto, las múltiples variables a optimizar se convierten en una sola. Mediante un ejemplo se muestra como la modificación propuesta ayuda a obtener distintos escenarios óptimos para considerar en la toma de decisiones.

Palabras clave: múltiples respuestas, optimización, metodología bayesiana, media geométrica ponderada

\section{Bayesian methodology for simultaneous optimization of multiple responses}

\begin{abstract}
The application of a bayesian methodology for simultaneous optimization of multiple responses is presented. A previous proposal is modified by introducing a modification that makes it more flexible, allowing working with the case in which not all the variables considered in the study are equally important. To incorporate in the methodology these differences related to the analyzed response variables the weighted geometric mean of the probabilities that each variable meets its own specification is used. With this change, the multiple optimization variables are converted into only one. An example to show how the proposed modification helps in achieving different optimal scenarios to consider in making decisions is discussed.
\end{abstract}

Keywords: multiple responses, optimization, bayesian methodology, weighted geometric mean 


\section{INTRODUCCIÓN}

Un problema común en el diseño de productos o procesos es la selección de niveles de los parámetros de un proceso para optimizar múltiples respuestas simultáneamente, a lo que se le conoce como problema de multirespuestas (Myers y Montgomery, 1995). A este respecto Noorossana y Ardakani (2010), mencionan que debido a que la calidad de los productos o procesos usualmente es evaluada mediante varias características de calidad o respuestas, se debiera de poner más atención en el diseño de parámetros con múltiples respuestas, para mejorar la calidad de estas simultáneamente. A este respecto Limón et al., (2011) mencionan que las técnicas y métodos utilizados para la optimización y análisis de variables han tenido un comportamiento creciente durante las últimas décadas con la aplicación y adaptación de principios estadísticos, enfocados al análisis de datos con comportamiento multivariante.

Cuando se trabaja con múltiples respuestas, una alternativa es analizar cada respuesta de manera separada, pero como mencionan Chiao y Hamada (2001), esto no es muy satisfactorio, especialmente cuando las respuestas están correlacionadas, ya que por ejemplo, análisis por separado pueden llevar a distintas recomendaciones en cuanto a los niveles de los factores importantes, aún más, cuando se ignoran las correlaciones ente las respuestas se puede perder la oportunidad de encontrar niveles de los factores que simultáneamente mejoren la calidad de todas las respuestas. Para abordar esta situación de la optimización simultánea de las respuestas se han propuesto varias técnicas con diferentes niveles de complejidad y algunas más completas respecto a los aspectos importantes que se deben considerar, entre las cuales se pueden encontrar las siguientes: función de deseabilidad, función de pérdida, e índices de capacidad.

Función de deseabilidad: Derringer y Suich (1980) proponen utilizar funciones de deseabilidad las cuales convierten el problema de múltiples respuestas, en un problema de una sola respuesta; esto es, la respuesta que se analiza es la deseabilidad total (D). Sin embargo este enfoque según Chiao y Hamada (2001) tiene dos desventajas: primero, $D$ puede ser difícil de modelar ya que es una función compleja de las $p$ respuestas; segundo, es difícil decir que es lo que significa la diferencia entre valores esperados de $D$, excepto que el mayor es el mejor. Otra desventaja de este enfoque es según Ko et al., (2005) el que no considera la estructura de varianzascovarianzas de las respuestas y según ellos ignorar esta información puede llevar a una solución no real, si en realidad las respuestas tienen varianzas significativamente diferentes o si están altamente correlacionadas. Se han propuesto otros enfoques basados en la deseabilidad para corregir algunos detalles de la propuesta original, como en Ch'ng et al., (2005) y Del Castillo et al., (1996).

Función de pérdida: Ames et al., (1997) proponen las funciones de pérdida para optimizar múltiples características de calidad. Mencionan que las funciones de pérdida proveen un marco conceptual que permite combinar criterios diferentes en una función objetivo única. Una función de pérdida describe la pérdida económica de la sociedad, provocada por las desviaciones de las características con respecto a su valor objetivo, debida a variaciones aleatorias y errores sistemáticos. La pérdida total a la sociedad debida a las desviaciones en todas las características del producto se puede tomar como la suma de las pérdidas debidas a las características individuales. Estas funciones de pérdida se deben minimizar para obtener las mejores condiciones de operación del proceso ya que esto minimizaría la pérdida del cliente o de la sociedad. Según Ames et al., (1997), al minimizar la función de pérdida además de la calidad más alta, también se produce la mayor estabilidad o robustez en el proceso contra las variaciones en la calidad debida a las variables de entrada.

Peterson (2004) menciona que actualmente existen enfoques basados en la función cuadrática de pérdida que consideran la estructura de correlaciones entre los diferentes tipos de respuestas, sin embargo, también menciona que esta técnica tiene dos inconvenientes serios, primero, no considera la incertidumbre de la matriz de varianzas-covarianzas de los errores en los modelos de regresión y segundo, en algunas ocasiones la función cuadrática de pérdida, especialmente alguna que se base en una forma cuadrática multivariada puede ser difícil de entender para algunos investigadores. 
Índices de capacidad: En estas técnicas se utiliza también la reducción de dimensionalidad por pasar el problema de optimizar múltiples respuestas, al problema de optimizar una sola, la cual involucra alguna operación con los índices de capacidad individuales calculados para cada respuesta en cada condición de operación del proceso

Entre los autores que sugieren las técnicas que utilizan estos índices de capacidad, se encuentran Ch'ng et al., (2005) que proponen utilizar el Cpm Total. Para optimizar el proceso de acuerdo a este índice propuesto se evalúan diferentes opciones de operación del proceso mediante el método del gradiente reducido generalizado a partir de un vector de valores iniciales, buscando maximizar el Cpm total según reportan en su trabajo. Otra alternativa es hacer programas que busquen en todas las combinaciones posibles de las variables la que sería la óptima con la desventaja de que el tiempo de procesamiento sería más lento, pero con la ventaja de que no arrojaría óptimos locales. Esta técnica además de que no considera la correlación entre las variables de respuesta, ni la incertidumbre en los parámetros de los modelos construidos, puede llevar a seleccionar como la condición de operación del proceso óptima alguna en la que uno o unos de los índices sean demasiado grandes para algunas respuestas, sin importar que las otras tomen valores demasiado bajos o inclusive que su media caiga fuera de especificaciones.

Plante (2001) propone los índices de capacidad del proceso MCpk y MCpm como criterios de optimización múltiple. Empezando por el índice de capacidad del proceso MCpk, este es igual a la media geométrica de las medidas de comportamiento Cpk individuales y según el autor, la filosofía fundamental detrás del índice MCpk tiene profundas implicaciones en la calidad, esto es, si una o más de las medidas de desempeño es inadecuada (índice cercano a cero), entonces el producto completo se vuelve inaceptable, sin importar los índices de capacidad de las restantes medidas de desempeño. De hecho el índice MCpk es fuertemente influenciado por las características de calidad que tienen una capacidad baja. Además este criterio es más universalmente aplicable, puesto que no requiere de un valor objetivo para cada medida de desempeño. El índice MCpk es una variable del tipo lo mayor es lo mejor, por lo que debe maximizarse, lo cual puede lograrse con el uso de métodos numéricos, por ejemplo el método del gradiente reducido generalizado que es el que utiliza el autor.

Similar al índice MCpk, el índice MCpm se calcula como la media geométrica de las medidas de desempeño Cpm individuales. La optimización es similar a la del MCpk, sin embargo el índice $M C p m$, como el índice Cpm, requiere de valores objetivo para cada característica de calidad, por lo que no es tan flexible con el índice MCpk. Plante (2001), reconoce que en algunas ocasiones no todas las características de calidad son igual de importantes, por lo que según dice puede ser más importante lograr mayores índices de capacidad en una o más características a expensas de algunas otras. Es por esto que en su enfoque se considera la posibilidad de considerar la importancia relativa de las diferentes características de calidad.

Estos dos enfoques tampoco consideran la correlación entre las variables de respuesta, ni la incertidumbre en los parámetros de los modelos construidos, y un inconveniente al utilizar el MCpk es que si en alguna condición de operación del proceso la media de alguna de las variables de respuesta cae fuera de sus especificaciones su Cpk será negativo y si la cantidad de variables a optimizar es $p$, donde $p$ es par no será posible calcular su MCpk, lo que provoca una inconsistencia en el método.

\section{Métodos que Maximizan la Probabilidad de Conformancia}

Según mencionan Chiao y Hamada (2001), cuando se analizan datos de múltiples respuestas, analizarlas por separado no ofrece resultados satisfactorios, especialmente cuando estas variables están correlacionadas. El realizar análisis separados puede llevar a conclusiones contradictorias respecto a los niveles de los factores importantes, ya que ciertos niveles de las variables pueden mejorar la calidad de alguna respuesta, pero empeorar la de otras. En el método que proponen se considera la matriz de varianzas-covarianzas dependiente de los factores experimentales y el criterio de decisión es la probabilidad de que todas las respuestas satisfagan simultáneamente sus respectivas especificaciones, por lo que la configuración óptima del proceso 
será aquella que maximice la probabilidad de que las variables de respuesta estén en la región $A$, lo cual se representa en la ecuación (1):

$P(Y \in A)$

Según estos autores la proporción de conformancia se determina por las medias, varianzas y correlaciones $(\rho)$ de la distribución multivariada de las respuestas, proponen construir modelos para estos parámetros, con los cuales se obtienen diferentes valores de los parámetros para diferentes niveles de los factores de interés, los cuales se sustituyen en la función de densidad de probabilidad conjunta $Y$, la cual se considera una normal multivariada $p$ dimensional con media $\mu=\left(\mu_{1}, \mu_{2}, \ldots, \mu_{p}\right)^{T}$ y matriz de varianza-covarianza $\Sigma$. La función de densidad de probabilidad conjunta $Y, f(Y ; \mu, \Sigma)$ está dada por la ecuación (2).

$f(Y ; \mu, \Sigma)=(2 \pi)^{-(m / 2)}|\Sigma|^{-1 / 2} x \exp \left[-\frac{1}{2}(Y-\mu)^{\prime} \Sigma^{-1}(Y-\mu)\right]$

Asumiendo que la distribución normal multivariada para $Y$ de la ecuación (2) es correcta, es posible evaluar la proporción de conformancia de la ecuación (1), para una $\Sigma$ y $\mu$ dadas, las cuales pueden ser estimadas con los modelos construidos previamente. Este enfoque no considera la incertidumbre en los parámetros estimados.

\section{El enfoque Bayesiano para Optimización de Respuestas Múltiples}

En el presente trabajo se utiliza un enfoque bayesiano propuesto por Peterson (2004), en el que también se considera la probabilidad de que el proceso cumpla con los requisitos de calidad de las características del producto a optimizar consideradas en el análisis y toma en cuenta la estructura de correlación y la incertidumbre en los parámetros de los modelos.

Miro-Quesada et al. (2004), mencionan que el problema de la optimización de respuestas múltiples consiste en escoger los valores de los $k$ factores controlables $x_{i}$, tal que el vector de respuestas $y$ tenga ciertas propiedades deseadas. Frecuentemente se presenta el caso en que estas propiedades son que el vector de respuestas $y$ satisfaga las especificaciones. Digamos que $A$ representa la región del espacio de respuestas definido por estas especificaciones. La región $A$ puede tener una forma arbitraria. Entonces la optimización de respuestas múltiples se puede resumir en un simple objetivo, el cual consiste en maximizar la probabilidad de tener el vector de respuestas dentro de la región de especificación $A$, por ejemplo, maximizar la siguiente probabilidad de conformancia:

$\max p(x)=p(y \in A \mid x$, datos $) \max p(x)=p(y \in A \mid x$, datos $)$

sujetoa :

sujeto $a$ :

$x \in R$

$x \in R$

Donde $y=B^{\prime} z(x)+\varepsilon$ y $R$ es la región en donde el modelo es válido, la cual normalmente se toma como la región experimental definida por la matriz de diseño $X$.

Según Peterson (2004) partiendo del modelo típico de regresión multivariada:

$Y=B z(x)+e$

Donde $B$ es una matriz $p x q$ de los coeficientes de regresión y $z(x)$ es el vector $q x 1$ de valores $x$ en los que se evalúa la ecuación (4). Además el vector e tiene una distribución normal multivariada con vector de medias igual a $\mathbf{0}$ y matriz de varianzas-covarianzas $\Sigma$. Para considerar la incertidumbre en los parámetros del modelo $B$ y $\Sigma$ se puede usar la densidad posterior predictiva 
$f(y \mid x$,datos). Utilizando la distribución previa conjunta no informativa clásica para $B$ y $\Sigma$ y el modelo de la ecuación (4), la densidad predictiva bayesiana para y se puede obtener en forma cerrada. La densidad predictiva bayesiana para el vector de valores $x$ en los que se evaluará la función, está dada por una distribución $t$ multivariada con $v$ grados de libertad $(g l)$, de la siguiente manera:

$$
f(y \mid x, \text { datos })=\frac{\Gamma\left(\frac{v+p}{2}\right)}{(\pi v)^{p / 2} \Gamma\left(\frac{v}{2}\right)} \sqrt{|H|}\left\{1+\frac{1}{v}\left(y-\hat{B}^{\prime} z(x)\right)^{\prime} H\left(y-\hat{B}^{\prime} z(x)\right)\right\}^{-\left(\frac{v+p}{2}\right)}
$$

Donde:

$$
H=\left(\frac{v}{N-q}\right) \frac{\hat{\Sigma}^{-1}}{1+z(x)^{\prime}\left(X^{\prime} X\right)^{-1} z(x)}
$$

Aquí $p$ es la cantidad de respuestas a optimizar, $X$ es la matriz de $q x N$ formada por los $N z\left(X_{i}\right)$ vectores de covariables, $\hat{B}$ es la estimación por mínimos cuadrados de $B, \Gamma($.$) se refiere a la$ función gamma, $\hat{\Sigma}$ es el estimador usual de $\Sigma$ calculado con los residuales del ajuste de la regresión multivariada, $v=N-p-q+1$ y $N$ es el tamaño de muestra.

Según Peterson (2004), debido a que la ecuación (5), sigue una distribución $t$ multivariada, es fácil simular valores para $y$ de esta densidad predictiva. Johnson (1987) en su libro Multivariate statistical simulation menciona que se puede simular una variable aleatoria $t$ - variada $y$, mediante la simulación de una variable aleatoria normal multivariada y una variable aleatoria chi-cuadrada independiente (Peterson, 2004). Si $W$ es una variable aleatoria normal con vector de medias igual a cero y una matriz de varianzas-covarianzas igual a $H^{-1}$ y además $U$ es una variable aleatoria chicuadrada que es independiente de $W$, entonces:

$y_{j}=\left(\sqrt{v} W_{j} / \sqrt{U}\right)+\hat{\mu}_{j} \quad$ para $\mathrm{j}=1, \ldots, \mathrm{p}$

Donde $y_{j}$ es el $j$-ésimo elemento de $y, W_{j}$ es el j-ésimo elemento de $W$ y $\widehat{\mu}_{j}$ es el $j$-ésimo elemento de $\widehat{\mu}=\widehat{B} z(x)$. Con lo anterior según Peterson (2004), se puede decir que $y$ sigue una distribución $t$ multivariada con $v$ grados de libertad.

Entonces la idea es generar una gran cantidad de valores de las respuestas utilizando la ecuación (6) y compararlos contra sus respectivas especificaciones para poder determinar la probabilidad de conformancia de cada una de las variables en cada condición de operación del proceso $z(x)$, para lo que se generó un programa en matlab, en el cual se incluyó un contador que se incrementa cada vez que el vector de las $p$ variables generadas cumplen con sus respectivas especificaciones, posteriormente este contador se divide entre la cantidad de iteraciones realizadas y así se obtienen las probabilidades para cada condición $\mathrm{z}(\mathrm{x})$.

Mientras que Peterson (2004) y Miró-Quesada et al., (2004) consideran como requisito que todas las variables cumplan con sus especificaciones de manera conjunta en cada iteración para incrementar el contador, en este trabajo lo que se propone es llevar contadores independientes para cada una de las respuestas de tal forma que al generar un vector de valores en cada iteración, cada respuesta es comparada con su respectiva especificación con lo que algunos 
contadores podrán incrementarse, mientras que otros no, dependiendo de si se cumple con los requisitos y de esta manera es posible calcular probabilidades de conformancia independientes dividiendo cada contador entre el número de iteraciones realizadas. Son estas probabilidades individuales las que permiten introducir diferentes ponderaciones o pesos para cada variable, de acuerdo con la importancia que tienen en el estudio mediante el cálculo de la media geométrica ponderada, con lo que la metodología se vuelve más flexible ya que permite abordar tanto el caso en el que todas las variables de respuesta son igual de importantes, como el caso en que existen diferencias entre ellas. Adicionalmente el llevar a cabo la optimización utilizando diferentes combinaciones de pesos en las variables de respuesta, permite generar diferentes escenarios que le den una visión más amplia a quien esté llevando a cabo la mejora a la hora de tomar una decisión.

\section{METODOLOGÍA}

A continuación se ilustra la metodología con las modificaciones propuestas y sus efectos en los resultados obtenidos mediante un ejemplo. La metodología propuesta, consta de las siguientes etapas:

Etapa I: Identificar las variables de control y de ruido, así como las variables de respuesta que se considerarán en el estudio.

Etapa II: Planear y realizar el experimento de tal forma que permita ajustar modelos para las diferentes variables de respuesta a optimizar.

Etapa III: Ajustar modelos de regresión multivariada utilizando mínimos cuadrados ordinarios y verificar que tengan un ajuste adecuado $\left(R^{2}>70 \%\right)$. Si alguna variable de respuesta no cumple con este requisito se saca del estudio.

Etapa IV: Determinar la cantidad de combinaciones de los factores de control a simularse, de tal forma que resulte una malla lo suficientemente fina que permita ajustar el modelo logístico y generar las iteraciones necesarias para cada corrida.

Etapa V: Cálculo de las probabilidades individuales y de la Mgp con las ponderaciones que se consideren adecuadas.

Etapa VI: Ajustar el modelo logístico para la Mgp. En este caso se utilizó minitab 15.

Etapa VII: Construir gráficos para comparar los resultados según la simulación y según el modelo logit. En este caso esto se hizo con la ayuda de Excel 2007. Si el modelo ajusta de manera adecuada a los datos simulados, el comportamiento de ambas gráficas debiera ser muy parecido. Previamente se verifica la significancia de los términos incluidos en el modelo según el software utilizado.

Etapa VIII: Optimizar (maximizar) el modelo logístico resultante utilizando el paquete Solver de Excel, iniciando la búsqueda en diferentes valores.

Etapa IX: Una vez que se ha seleccionado la condición óptima de operación del proceso según el paquete Solver, realizar una simulación lo suficientemente grande con el programa de matlab y calcular según el modelo logístico en esa condición la media geométrica ponderada de las probabilidades y comparar.

Etapa $X$ : Concluir.

\section{CASO DE ESTUDIO}

Fases I y II: Para ilustrar la metodología anterior se toman los datos del problema propuesto por Myers y Montgomery (1995), en el cual se consideran tres variables de diseño, tiempo de reacción $\left(x_{1}\right)$, temperatura $\left(x_{2}\right)$ y porcentaje de catalizador $\left(x_{3}\right)$ y dos variables de respuesta, porcentaje de 
conversión $\left(y_{1}\right)$ y actividad térmica $\left(y_{2}\right)$ y se utiliza un diseño central compuesto con seis corridas centrales. En este caso se consideró a $\mathrm{x}_{1}$ como variable de ruido $\left(\mathrm{x}_{\mathrm{r}}\right)$, por lo que la optimización se realizará con respecto a las variables de control $\left(x_{c}\right) x_{2}$ y $x_{3}$. Los datos se muestran en la tabla 1.

Fase III: Con los datos de este experimento el modelo de segundo orden ajustado para la variable conversión es el siguiente:

$y_{1}=81.1+1.03 x_{1}+4.04 x_{2}+6.20 x_{3}-1.83 x_{1}^{2}+2.94 x_{2}^{2}-5.19 x_{3}^{2}+2.12 x_{1} x_{2}+11.4 x_{1} x_{3}$

$-3.88 x_{2} x_{3}$

Con $\mathrm{R}^{2}=92.0 \%$.

Mientras que el modelo de segundo orden ajustado para la variable actividad es el siguiente:

$y_{2}=59.8+3.58 x_{1}+0.255 x_{2}+2.23 x_{3}+0.835 x_{1}^{2}+0.075 x_{2}^{2}+0.057 x_{3}^{2}-0.388 x_{1} x_{2}-0.038 x_{1} x_{3}$

$+0.312 x_{2} x_{3}$

Con $\mathrm{R}^{2}=89.2 \%$

Tabla 1: Datos tomados para ilustrar la metodología

\begin{tabular}{ccccc}
\hline $\mathrm{x}_{1}$ & $\mathrm{x}_{2}$ & $\begin{array}{c}\mathrm{x}_{3} \\
\text { Catalizador }\end{array}$ & $\begin{array}{c}\mathrm{y}_{1} \\
\text { Conversión }\end{array}$ & $\begin{array}{c}y_{2} \\
\text { Actividad }\end{array}$ \\
\hline-1.000 & -1.000 & -1.000 & 74 & 53.2 \\
1.000 & -1.000 & -1.000 & 51 & 62.9 \\
-1.000 & 1.000 & -1.000 & 88 & 53.4 \\
1.000 & 1.000 & -1.000 & 70 & 62.6 \\
-1.000 & -1.000 & 1.000 & 71 & 57.3 \\
1.000 & -1.000 & 1.000 & 90 & 67.9 \\
-1.000 & 1.000 & 1.000 & 66 & 59.8 \\
1.000 & 1.000 & 1.000 & 97 & 67.8 \\
-1.682 & 0.000 & 0.000 & 76 & 59.1 \\
1.682 & 0.000 & 0.000 & 79 & 65.9 \\
0.000 & -1.682 & 0.000 & 85 & 60.0 \\
0.000 & 1.682 & 0.000 & 97 & 60.7 \\
0.000 & 0.000 & -1.682 & 55 & 57.4 \\
0.000 & 0.000 & 1.682 & 81 & 63.2 \\
0.000 & 0.000 & 0.000 & 81 & 59.2 \\
0.000 & 0.000 & 0.000 & 75 & 60.4 \\
0.000 & 0.000 & 0.000 & 76 & 59.1 \\
0.000 & 0.000 & 0.000 & 83 & 60.6 \\
0.000 & 0.000 & 0.000 & 80 & 60.8 \\
0.000 & 0.000 & 0.000 & 91 & 58.9 \\
\hline
\end{tabular}

Debido a que en ambos modelos sus coeficientes de determinación fueron mayores a $70 \%$, la metodología de optimización puede ser aplicada a ambas variables de respuesta utilizando los modelos (7) y (8) en la ecuación (6) para la generación de valores. 
Fase IV: En cuanto a la cantidad de combinaciones a simular, en este caso fue un arreglo $11^{2}$, es decir, se trabajó con 121 combinaciones diferentes de los factores de control $x_{2} y x_{3}$, utilizando los siguientes niveles para cada variable:

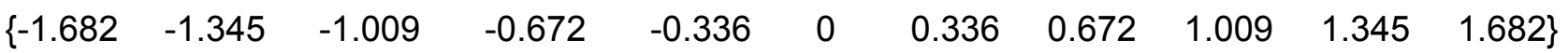

Es decir se consideró desde el nivel más pequeño para cada variable según se puede observar en la tabla 1, hasta su nivel más grande, con saltos de 0.3364 para que quedaran espacios de la misma dimensión entre cada nivel de las variables.

Una vez que se determinó la cantidad de combinaciones, se requiere determinar la cantidad de corridas a simularse en cada una de ellas utilizando la ecuación (6). En este caso se utilizaron 5000 corridas en cada una de las 121 combinaciones, es decir, 5000 vectores de dos respuestas cada uno $\left(y_{1}\right.$ y $\left.y_{2}\right)$, con las cuales se calculó la probabilidad de que cada respuesta cumpliera con su respectiva especificación, dividiendo la cantidad de variables simuladas que tomaron un valor aceptable entre el número de variables generadas. Es en este punto también en el que se atiende la robustez, debido a para cada una de las 121 combinaciones de $x_{2}$ y $x_{3}$, mientras estas permanecen fijas en la simulación de los 5000 vectores de respuestas, la variable $x_{1}$ toma un valor diferente en cada iteración, el cual se genera de una distribución normal $(0,0.1)$, con lo que se forma el vector de covariables $x_{1}, x_{2}$ y $x_{3}$, de tal forma que si de entre las 121 combinaciones existe alguna que de una probabilidad alta de conformancia, también se está asegurando de que esta combinación será robusta a cambios en la variable $\mathrm{x}_{1}$.

Fase V: Cuando se han generado las iteraciones necesarias se procede a hacer los cálculos de la media geométrica con las ponderaciones que se crean convenientes de acuerdo a la relevancia en la funcionalidad o costos del producto final de cada una de las variables de respuesta analizadas. En este ejemplo se consideraron cuatro escenarios distintos, en cada uno de los cuales las medias geométricas se calcularon con las ponderaciones que se indican en la tabla 2.

Tabla 2: Ponderaciones consideradas para las variables de respuesta

\begin{tabular}{ccccc}
\cline { 2 - 4 } & \multicolumn{4}{c}{ Ponderaciones } \\
\hline Variable & $M_{1}$ & $M_{1} p_{2}$ & $M_{1} p_{3}$ & $M g p_{4}$ \\
$y_{1}$ & 1 & 0.6 & 0.8 & 0.2 \\
$y_{2}$ & 1 & 0.4 & 0.2 & 0.8 \\
\hline
\end{tabular}

En la tabla 3 se muestran los resultados obtenidos para las últimas combinaciones simuladas, desde la 89 hasta la 121 , es decir, desde $x_{2}=1.0092$ hasta $x_{2}=1.682$ con los once niveles de $x_{3}$ ya que fue en esta zona en donde en todos los casos se encontraron los resultados óptimos. Los mejores resultados obtenidos en esta malla, así como sus condiciones de operación correspondientes se encuentran resumidos en la tabla 4. Posteriormente se mostrarán gráficamente los 121 resultados completos.

Para realizar los cálculos de las columnas $p_{1}$ y $p_{2}$ se consideraron las siguientes especificaciones para cada característica de calidad: $\left\{y_{1} \geq 80\right.$ y $\left.55 \leq y_{2} \leq 60\right\}$.

Por lo que cada vector generado se compara contra estos valores, para calcular las probabilidades de conformancia. En este punto también debe mencionarse que esta metodología de optimización funciona adecuadamente tanto para especificaciones bilaterales como unilaterales.

Fase VI: Toda vez que se ha simulado la malla con las diferentes combinaciones de los factores de interés, lo siguiente es ajustar un modelo logístico para Mgp. En las ecuaciones (9), (10), (11) y 
(12), se muestran los modelos ajustados para la $M g p_{1}, M g p_{2}, M g p_{3}$ y $M g p_{4}$ respectivamente considerando las variables de control, todos construidos utilizando el software minitab 15.

Tabla 3: Resultados obtenidos para las últimas combinaciones de la malla simulada

\begin{tabular}{|c|c|c|c|c|c|c|c|}
\hline$x_{2}$ & $x_{3}$ & $p_{1}$ & $p_{2}$ & $M g p_{1}$ & $M g p_{2}$ & $M g p_{3}$ & $\mathrm{Mgp}_{4}$ \\
\hline 1.0092 & -1.682 & 0.0946 & 0.5532 & 0.2288 & 0.1917 & 0.1347 & 0.3886 \\
\hline 1.0092 & -1.3456 & 0.2574 & 0.6788 & 0.4180 & 0.3794 & 0.3125 & 0.5591 \\
\hline 1.0092 & -1.0092 & 0.5268 & 0.7128 & 0.6128 & 0.5945 & 0.5596 & 0.6710 \\
\hline 1.0092 & -0.6728 & 0.7714 & 0.6750 & 0.7216 & 0.7313 & 0.7511 & 0.6933 \\
\hline 1.0092 & -0.3364 & 0.8780 & 0.5880 & 0.7185 & 0.7479 & 0.8103 & 0.6371 \\
\hline 1.0092 & 0 & 0.9192 & 0.4500 & 0.6431 & 0.6908 & 0.7968 & 0.5191 \\
\hline 1.0092 & 0.3364 & 0.9176 & 0.3036 & 0.5278 & 0.5895 & 0.7355 & 0.3788 \\
\hline 1.0092 & 0.6728 & 0.8824 & 0.1806 & 0.3992 & 0.4678 & 0.6425 & 0.2480 \\
\hline 1.0092 & 1.0092 & 0.7938 & 0.1146 & 0.3016 & 0.3660 & 0.5390 & 0.1688 \\
\hline 1.0092 & 1.3456 & 0.6018 & 0.0724 & 0.2087 & 0.2580 & 0.3940 & 0.1106 \\
\hline 1.0092 & 1.682 & 0.3720 & 0.0468 & 0.1319 & 0.1623 & 0.2457 & 0.0708 \\
\hline 1.3456 & -1.682 & 0.2920 & 0.5330 & 0.3945 & 0.3715 & 0.3293 & 0.4726 \\
\hline 1.3456 & -1.3456 & 0.5484 & 0.6370 & 0.5910 & 0.5823 & 0.5651 & 0.6182 \\
\hline 1.3456 & -1.0092 & 0.8026 & 0.6750 & 0.7360 & 0.7489 & 0.7753 & 0.6988 \\
\hline 1.3456 & -0.6728 & 0.9148 & 0.6576 & 0.7756 & 0.8016 & 0.8564 & 0.7025 \\
\hline 1.3456 & -0.3364 & 0.9468 & 0.5526 & 0.7233 & 0.7633 & 0.8501 & 0.6154 \\
\hline 1.3456 & 0 & 0.9676 & 0.4144 & 0.6332 & 0.6893 & 0.8167 & 0.4910 \\
\hline 1.3456 & 0.3364 & 0.9592 & 0.2852 & 0.5230 & 0.5905 & 0.7526 & 0.3635 \\
\hline 1.3456 & 0.6728 & 0.9322 & 0.1698 & 0.3979 & 0.4717 & 0.6631 & 0.2387 \\
\hline 1.3456 & 1.0092 & 0.8534 & 0.1162 & 0.3149 & 0.3844 & 0.5727 & 0.1731 \\
\hline 1.3456 & 1.3456 & 0.7006 & 0.0674 & 0.2173 & 0.2746 & 0.4386 & 0.1077 \\
\hline 1.3456 & 1.682 & 0.4410 & 0.0604 & 0.1632 & 0.1991 & 0.2963 & 0.0899 \\
\hline 1.682 & -1.682 & 0.5844 & 0.4950 & 0.5378 & 0.5468 & 0.5653 & 0.5117 \\
\hline 1.682 & -1.3456 & 0.8088 & 0.5794 & 0.6846 & 0.7078 & 0.7566 & 0.6194 \\
\hline 1.682 & -1.0092 & 0.9244 & 0.6252 & 0.7602 & 0.7905 & 0.8549 & 0.6761 \\
\hline 1.682 & -0.6728 & 0.9688 & 0.6184 & 0.7740 & 0.8096 & 0.8856 & 0.6765 \\
\hline 1.682 & -0.3364 & 0.9822 & 0.5132 & 0.7100 & 0.7576 & 0.8626 & 0.5843 \\
\hline 1.682 & 0 & 0.9804 & 0.3842 & 0.6137 & 0.6740 & 0.8129 & 0.4634 \\
\hline 1.682 & 0.3364 & 0.9794 & 0.2780 & 0.5218 & 0.5918 & 0.7613 & 0.3576 \\
\hline 1.682 & 0.6728 & 0.9580 & 0.1734 & 0.4076 & 0.4835 & 0.6806 & 0.2441 \\
\hline 1.682 & 1.0092 & 0.9120 & 0.1114 & 0.3187 & 0.3933 & 0.5989 & 0.1696 \\
\hline 1.682 & 1.3456 & 0.7654 & 0.0834 & 0.2527 & 0.3154 & 0.4913 & 0.1299 \\
\hline 1.682 & 1.682 & 0.5334 & 0.0598 & 0.1786 & 0.2223 & 0.3443 & 0.0926 \\
\hline
\end{tabular}

Tabla 4: Mejores resultados obtenidos en la malla simulada para cada ponderación

\begin{tabular}{lrcc}
\hline & \multicolumn{2}{c}{ Condición } & Mejor resultado \\
& \multicolumn{1}{c}{$\mathrm{x}_{2}$} & \multicolumn{1}{c}{$\mathrm{x}_{3}$} & (malla) \\
\hline $\mathrm{Mgp}_{1}$ & 1.3456 & -0.6728 & 0.7756 \\
$\mathrm{Mgp}_{2}$ & 1.682 & -0.6728 & 0.8096 \\
$\mathrm{Mgp}_{3}$ & 1.682 & -0.6728 & 0.8856 \\
$\mathrm{Mgp}_{4}$ & 1.3456 & -0.6728 & 0.7025 \\
\hline
\end{tabular}

$$
\begin{aligned}
& \log i t\left(\widehat{p}\left(x_{c}\right)\right)=0.123+0.398 x_{1}+0.009 x_{2}-1.078 x_{1} x_{2}+0.229 x_{1}^{2}-1.370 x_{2}^{2}-0.107 x_{1}^{2} x_{2} \\
& +0.514 x_{1} x_{2}^{2}+0.141 x_{1}^{2} x_{2}^{2}-0.159 x_{1}^{3}+0.376 x_{2}^{3}+0.139 x_{1}^{3} x_{2}+0.168 x_{1} x_{2}^{3}-0.025 x_{1}^{4} \\
& +0.176 x_{2}^{4}-0.054 x_{1}^{4} x_{2}-0.261 x_{1} x_{2}^{4}+0.014 x_{1}^{5}-0.071 x_{2}^{5}-0.007 x_{1}^{5} x_{2}-0.013 x_{1} x_{2}^{5} \\
& -0.006 x_{1}^{6}-0.011 x_{2}^{6}+0.018 x_{1}^{6} x_{2}+0.039 x_{1} x_{2}^{6}
\end{aligned}
$$


$\log \operatorname{it}\left(\hat{p}\left(x_{c}\right)\right)=0.158+0.523 x_{1}+0.316 x_{2}-1.204 x_{1} x_{2}+0.297 x_{1}^{2}-1.485 x_{2}^{2}-0.134 x_{1}^{2} x_{2}$

$+0.529 x_{1} x_{2}^{2}+0.155 x_{1}^{2} x_{2}^{2}-0.180 x_{1}^{3}+0.350 x_{2}^{3}+0.154 x_{1}^{3} x_{2}+0.201 x_{1} x_{2}^{3}-0.034 x_{1}^{4}$

$+0.187 x_{2}^{4}-0.054 x_{1}^{4} x_{2}-0.261 x_{1} x_{2}^{4}+0.014 x_{1}^{5}-0.070 x_{2}^{5}-0.008 x_{1}^{5} x_{2}-0.019 x_{1} x_{2}^{5}$

$-0.006 x_{1}^{6}-0.012 x_{2}^{6}+0.017 x_{1}^{6} x_{2}+0.038 x_{1} x_{2}^{6}$

$\log \operatorname{it}\left(\hat{p}\left(x_{c}\right)\right)=0.240+0.814 x_{1}+0.982 x_{2}-1.414 x_{1} x_{2}+0.479 x_{1}^{2}-1.651 x_{2}^{2}-0.178 x_{1}^{2} x_{2}$

$+0.457 x_{1} x_{2}^{2}+0.168 x_{1}^{2} x_{2}^{2}-0.206 x_{1}^{3}+0.264 x_{2}^{3}+0.166 x_{1}^{3} x_{2}+0.254 x_{1} x_{2}^{3}-0.054 x_{1}^{4}$

$+0.167 x_{2}^{4}-0.049 x_{1}^{4} x_{2}-0.210 x_{1} x_{2}^{4}+0.012 x_{1}^{5}-0.064 x_{2}^{5}-0.008 x_{1}^{5} x_{2}-0.029 x_{1} x_{2}^{5}$

$-0.005 x_{1}^{6}-0.006 x_{2}^{6}+0.014 x_{1}^{6} x_{2}+0.027 x_{1} x_{2}^{6}$

$\log \operatorname{it}\left(\hat{p}\left(x_{c}\right)\right)=0.045+0.065 x_{1}-0.914 x_{2}-0.659 x_{1} x_{2}+0.063 x_{1}^{2}-0.886 x_{2}^{2}-0.018 x_{1}^{2} x_{2}$

$+0.335 x_{1} x_{2}^{2}+0.088 x_{1}^{2} x_{2}^{2}-0.089 x_{1}^{3}+0.419 x_{2}^{3}+0.091 x_{1}^{3} x_{2}+0.064 x_{1} x_{2}^{3}-0.011 x_{1}^{4}$

$+0.059 x_{2}^{4}-0.035 x_{1}^{4} x_{2}-0.187 x_{1} x_{2}^{4}+0.012 x_{1}^{5}-0.065 x_{2}^{5}-0.006 x_{1}^{5} x_{2}+0.005 x_{1} x_{2}^{5}$

$-0.005 x_{1}^{6}+0.007 x_{2}^{6}+0.014 x_{1}^{6} x_{2}+0.029 x_{1} x_{2}^{6}$

Fase VII: Para apreciar el ajuste de los modelos a cada una de las medias geométricas para la que fue ajustado, se procedió a construir gráficos en Excel en donde se representaran al mismo tiempo las medias geométricas de las probabilidades de conformancia según las 5000 simulaciones hechas con matlab para cada una de las 121 combinaciones de los factores de interés según la ecuación (6) y las medias geométricas pronosticadas por los modelos anteriores en estas mismas combinaciones. Las comparaciones de los resultados simulados contra los pronosticados por el modelo para la $M g p_{1}, M g p_{2}, M g p_{3}$ y $M g p_{4}$ se muestran en las figuras 1, 2, 3 y 4 , respectivamente. Como puede verse los modelos pronostican con mucha exactitud lo obtenido mediante la simulación ya que el valor pronosticado prácticamente se sobrepone al valor simulado, por lo cual estos modelos pueden utilizarse para la optimización mediante métodos numéricos. En estas figuras también puede apreciarse en donde se encuentran los valores más altos para cada una de las medias geométricas ponderadas y por lo tanto dan una aproximación de en donde se ubicarán las zonas óptimas.

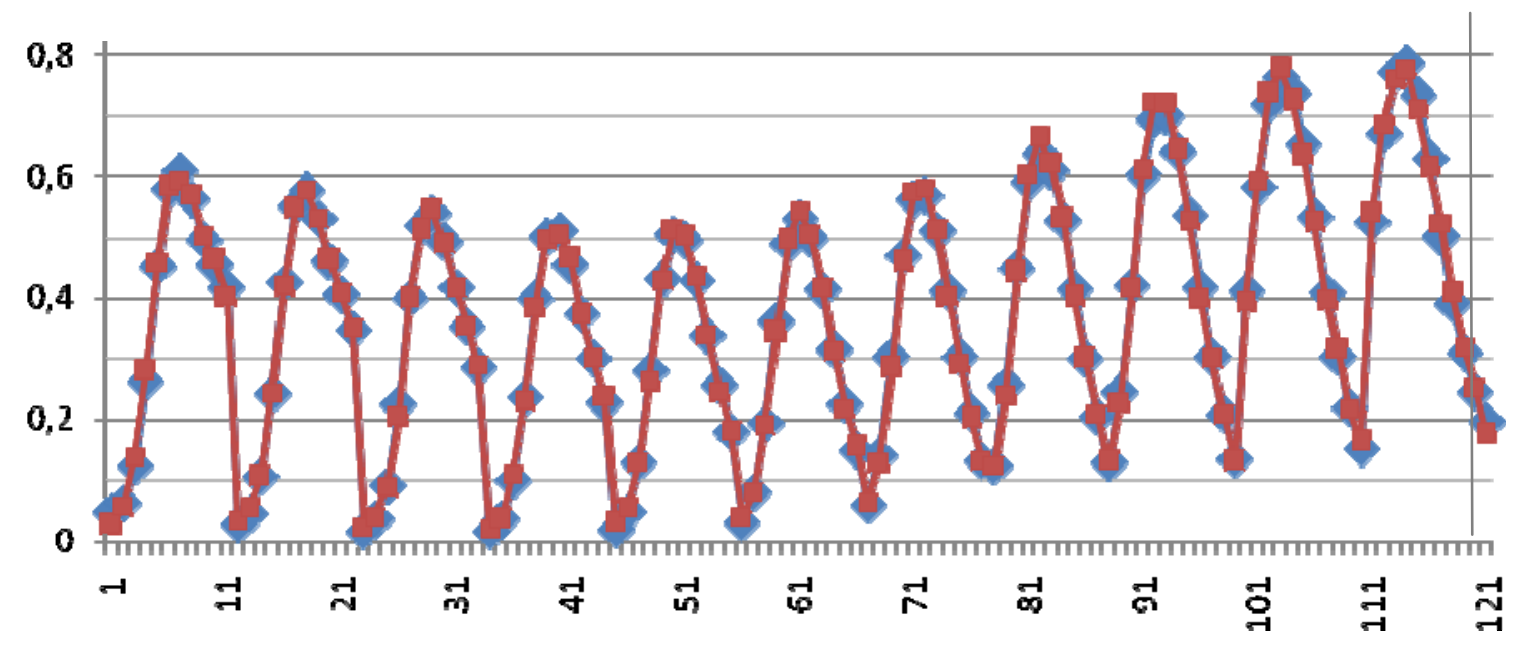

Fig. 1: Comparación de los resultados simulados contra los pronosticados para $\mathrm{Mgp}_{1}$. En la figura: $\checkmark$ son los resultados del modelo y $\boldsymbol{\nabla}$ los resultados de la simulación. 


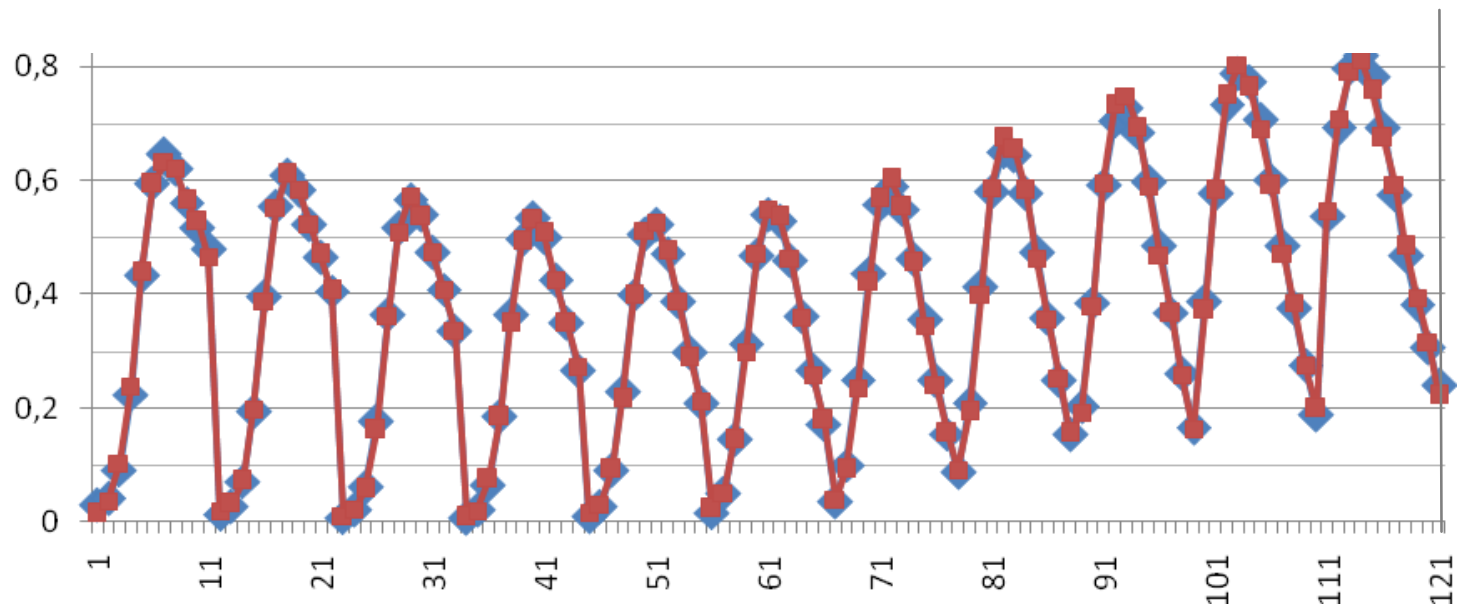

Fig. 2: Comparación de los resultados simulados contra los pronosticados para $\mathrm{Mgp}_{2}$. En la figura: $\checkmark$ son los resultados del modelo y $\square$ los resultados de la simulación.

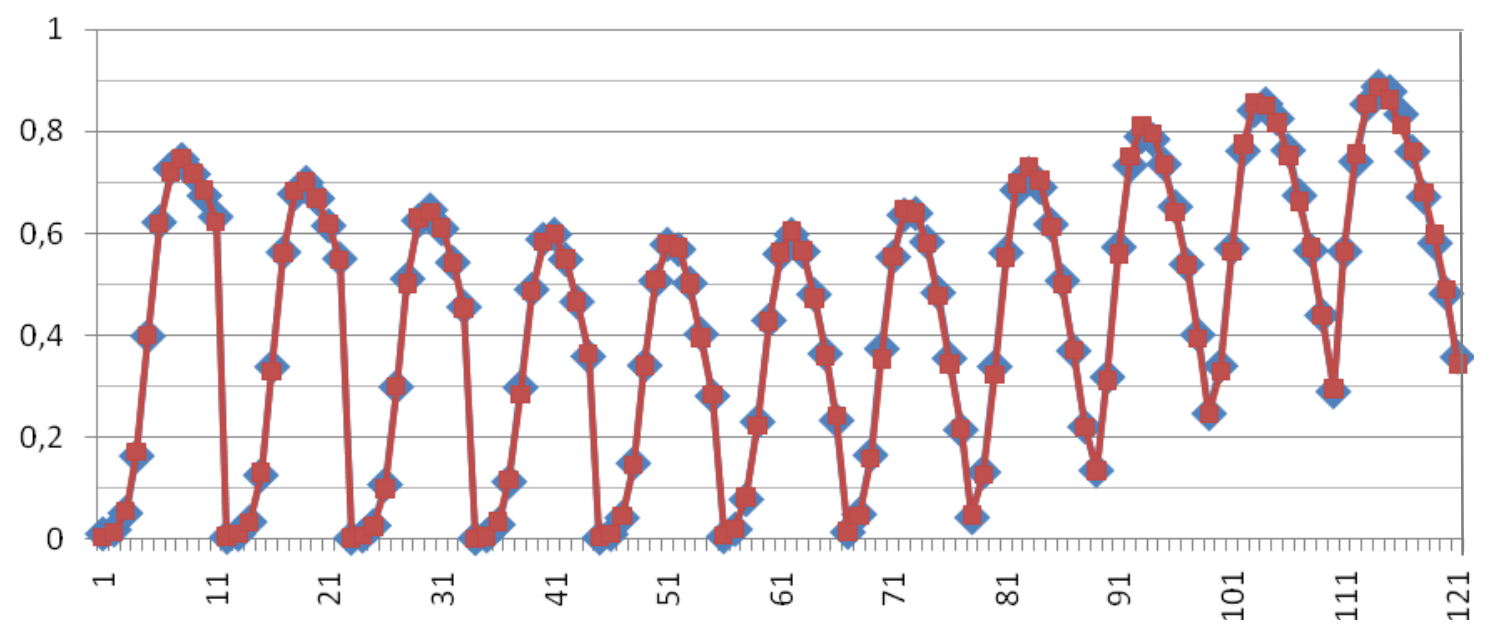

Fig. 3: Comparación de los resultados simulados contra los pronosticados para $\mathrm{Mgp}_{3}$. En la figura: $\checkmark$ son los resultados del modelo y $\square$ los resultados de la simulación.

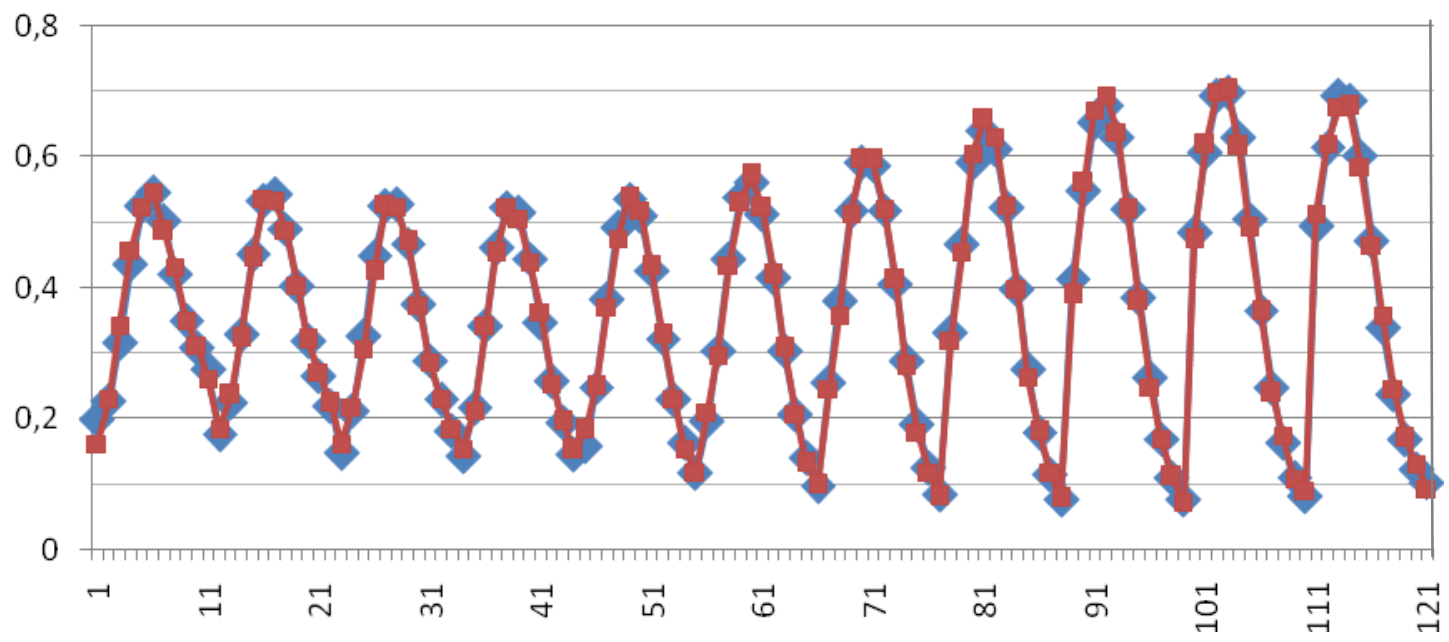

Fig. 4: Comparación de los resultados simulados contra los pronosticados para $\mathrm{Mgp}_{4}$. En la figura: $\checkmark$ son los resultados del modelo y $\boldsymbol{\square}$ los resultados de la simulación. 


\section{RESULTADOS}

Fase VIII: Después de verificar gráficamente que los modelos realmente representen los resultados simulados, así como su significancia, se puede proceder a la fase de optimización con ellos, para la cual se utilizó el método del gradiente reducido generalizado, el cual se puede encontrar en el paquete Solver de Excel, lo que lo hace muy accesible a quien desee utilizarlo. Este algoritmo de optimización permite incorporar restricciones en las zonas de búsqueda, lo que proporciona una ventaja, ya que de esta manera podremos asegurarnos de que la solución propuesta estará dentro de la región en la que se diseñó el experimento.

A la hora de utilizar el Solver de Excel, es necesario empezar el algoritmo con valores iniciales para las variables de interés. Se sugiere que se inicie el algoritmo en varias ocasiones con distintos valores para evaluar la consistencia de la solución alcanzada. En este caso se utilizaron 15 valores iniciales diferentes para la búsqueda de los valores óptimos, estos valores de partida fueron los mismos para los cuatro modelos logísticos. Los resultados obtenidos muestran que en los cuatro casos siempre hubo convergencia hacia el mismo resultado, independientemente de los valores iniciales, lo que puede apreciarse en la tabla 5.

Tabla 5: Resultados de la optimización

\begin{tabular}{|cc|cc|cc|cc|cc|}
\hline \multicolumn{2}{|c|}{ Valores iniciales } & \multicolumn{6}{|c|}{ Resultados óptimos encontrados para las variables $\mathrm{x}_{2} \mathrm{y}_{3}$} \\
\hline $\mathrm{X}_{2}$ & $\mathrm{X}_{3}$ & \multicolumn{2}{|c|}{$M g p_{1}$} & \multicolumn{2}{|c|}{$M g p_{2}$} & \multicolumn{2}{|c|}{$M g p_{3}$} & \multicolumn{3}{c|}{$M g p_{4}$} \\
0.40 & -0.40 & 1.6633 & -0.7668 & 1.682 & -0.7307 & 1.682 & -0.5969 & 1.4817 & -0.848 \\
0.20 & -1.00 & 1.6633 & -0.7668 & 1.682 & -0.7307 & 1.682 & -0.5969 & 1.4817 & -0.848 \\
0.70 & 0.70 & 1.6633 & -0.7668 & 1.682 & -0.7307 & 1.682 & -0.5969 & 1.4817 & -0.848 \\
-0.30 & -0.70 & 1.6633 & -0.7668 & 1.682 & -0.7307 & 1.682 & -0.5969 & 1.4817 & -0.848 \\
0.90 & -0.90 & 1.6633 & -0.7668 & 1.682 & -0.7307 & 1.682 & -0.5969 & 1.4817 & -0.848 \\
0.80 & 0.25 & 1.6633 & -0.7668 & 1.682 & -0.7307 & 1.682 & -0.5969 & 1.4817 & -0.848 \\
0.00 & 0.00 & 1.6633 & -0.7668 & 1.682 & -0.7307 & 1.682 & -0.5969 & 1.4817 & -0.848 \\
0.50 & -0.50 & 1.6633 & -0.7668 & 1.682 & -0.7307 & 1.682 & -0.5969 & 1.4817 & -0.848 \\
0.30 & 0.50 & 1.6633 & -0.7668 & 1.682 & -0.7307 & 1.682 & -0.5969 & 1.4817 & -0.848 \\
0.25 & 0.25 & 1.6633 & -0.7668 & 1.682 & -0.7307 & 1.682 & -0.5969 & 1.4817 & -0.848 \\
0.47 & 0.47 & 1.6633 & -0.7668 & 1.682 & -0.7307 & 1.682 & -0.5969 & 1.4817 & -0.848 \\
0.94 & 0.80 & 1.6633 & -0.7668 & 1.682 & -0.7307 & 1.682 & -0.5969 & 1.4817 & -0.848 \\
1.50 & -1.50 & 1.6633 & -0.7668 & 1.682 & -0.7307 & 1.682 & -0.5969 & 1.4817 & -0.848 \\
-0.10 & 0.10 & 1.6633 & -0.7668 & 1.682 & -0.7307 & 1.682 & -0.5969 & 1.4817 & -0.848 \\
1.60 & 1.60 & 1.6633 & -0.7668 & 1.682 & -0.7307 & 1.682 & -0.5969 & 1.4817 & -0.848 \\
\hline
\end{tabular}

Fase IX: Una vez obtenidas las condiciones óptimas de operación para el proceso, se procede a hacer en cada condición de operación propuesta para cada una de las medias geométricas ponderadas analizadas, una evaluación de los resultados, primeramente sustituyendo estos valores en sus respectivos modelos logísticos y posteriormente haciendo una simulación como corrida de confirmación de 100000 nuevos vectores de respuestas. Si la metodología propuesta trabaja de forma adecuada los valores alcanzados en la evaluación tanto por el modelo como por la simulación deberán ser similares y deberán también ser valores similares en magnitud o más altos al compararlos con los obtenidos al realizar la malla en las 121 combinaciones. Esta comparación entre las mayores medias geométricas ponderadas obtenidas en la malla realizada con 5000 simulaciones (véase tabla 4), las obtenidas en la simulación con 100000 valores y las pronosticadas según el modelo logístico, estas dos últimas para validar las condiciones óptimas 
propuestas en la optimización, se muestran en la tabla 6 , en la cual puede apreciarse que los resultados obtenidos en las condiciones óptimas propuestas, según los modelos y la simulación son muy similares a las ya obtenidas en la malla simulada al inicio, lo que indica que los resultados obtenidos desde el principio ya eran los suficientemente buenos y quedaba poco espacio para la mejora, lo que pudiera no siempre suceder.

La tabla 7 muestra desglosados los resultados obtenidos con los 100000 vectores simulados como validación para $M g p_{1}, M g p_{2}, M g p_{3}$ y $M g p_{4}$, en la cual, la columna $M g p$ se refiere a las medias geométricas ponderadas calculadas utilizando las ponderaciones descritas en la tabla 2 partiendo de los valores $p_{1}$ y $p_{2}$ de las columnas anteriores. En cuanto a las columnas Media $y_{1}$ y Media $y_{2}$ estas se refieren a las medias calculadas con los 100000 valores generados durante la validación para $y_{1}$ y $y_{2}$ respectivamente.

Tabla 6: Medias geométricas ponderadas más altas obtenidas según el modelo y las simulaciones

\begin{tabular}{lccc}
\hline & $\begin{array}{c}\text { Mgp } \\
\text { Modelo }\end{array}$ & $\begin{array}{c}\text { Mgp Simulación } \\
\text { (Validación, 100000) }\end{array}$ & $\begin{array}{c}\text { Mgp Simulación } \\
\text { (malla 5000) }\end{array}$ \\
\hline$M g p_{1}$ & 0.7887 & 0.7714 & 0.7756 \\
$M g p_{2}$ & 0.8204 & 0.8075 & 0.8096 \\
$M g p_{3}$ & 0.8890 & 0.8821 & 0.8856 \\
$M g p_{4}$ & 0.7086 & 0.7042 & 0.7025 \\
\hline
\end{tabular}

Tabla 7: Resultados de la evaluación por simulación de las condiciones óptimas encontradas

\begin{tabular}{cccccccc}
\hline & \multicolumn{2}{c}{ Variables } & \multicolumn{3}{c}{ Probabilidades } & Media & Media \\
& $\mathrm{x}_{2}$ & $\mathrm{x}_{3}$ & $\mathrm{p}_{1}$ & $\mathrm{p}_{2}$ & Mgp & $y_{1}$ & $\mathrm{y}_{2}$ \\
\hline $\mathrm{Mgp}_{1}$ & 1.6630 & -0.7670 & 0.9581 & 0.6211 & 0.7714 & 93.05 & 58.44 \\
$\mathrm{Mgp}_{2}$ & 1.6820 & -0.7310 & 0.9638 & 0.6192 & 0.8075 & 93.63 & 58.51 \\
$\mathrm{Mgp}_{3}$ & 1.6820 & -0.5970 & 0.9730 & 0.5959 & 0.8821 & 94.57 & 58.86 \\
$\mathrm{Mgp}_{4}$ & 1.4817 & -0.8480 & 0.9138 & 0.6598 & 0.7042 & 89.42 & 58.14 \\
\hline
\end{tabular}

Conforme a los valores de la tabla 7 , se puede observar que los resultados de $M g p_{2}$ referentes a $y_{1}$ son mejores que los reportados en $M g p_{1}$ para esta misma variable de respuesta, contrario a lo que sucede con $y_{2}$ que son peores, es decir, $p_{1}$ de $M g p_{2}$ es mayor que $p_{1}$ de $M g p_{1}$, mientras que con $p_{2}$ sucede lo inverso, así también, si se revisan sus medias calculadas se puede ver que la media para $y_{1}$ de $M g p_{2}$ se aleja más de 80 que es su límite inferior de especificación con respecto a su media en $M g p_{1}$ y por otra parte la media para $y_{2}$ de $M g p_{2}$ se aleja más de su valor objetivo, o centro de la especificación que es 57.5 comparada con la media para $y_{2}$ de $M g p_{1}$. Esto se debe a que en $\mathrm{Mgp}_{2}$ se le da un mayor peso a $\mathrm{y}_{1}(0.6)$ que a $\mathrm{y}_{2}(0.4)$, lo que provoca que la solución óptima se desplace hacia donde se comporte mejor $y_{1}$ a expensas del resultado de $y_{2}$. Continuando con el análisis de la tabla 7 , si se observan los resultados de $M g p_{3}$ se pueden ver aun más marcados los cambios a favor de $y_{1}$ y en contra de $y_{2}$ si se compara con $M g p_{1}$, esto debido a que se le da un peso aun mayor a $y_{1}(0.8)$ contra el que se le da a $y_{2}(0.2)$, por lo que cuando se observan los valores promedios se puede ver que $y_{1}$ se aleja más de 80 , lo que es deseable, mientras que $y_{2}$ también se aleja más de su valor objetivo, lo que no es deseable, pero se puede permitir, porque según las ponderaciones empleadas esta variable es menos importante. El efecto contrario respecto a $y_{1}$ y $y_{2}$ puede observarse cuando se revisan los resultados de $\mathrm{Mgp}_{4}$, ya que en este caso se busca un mejor resultado en $y_{2}$ a expensas de $y_{1}$, lo que queda de manifiesto en las ponderaciones otorgadas de 0.8 y 0.2 respectivamente, con lo cual se beneficia a $p_{2}$ mostrando su valor más alto de los cuatro casos analizados, y se castiga a $p_{1}$ que muestra su valor más bajo, de igual forma se aprecia que la media de $y_{2}$ se acerca más a su valor objetivo, mientras que la media de $y_{1}$ se acerca más a su límite inferior de especificación. 


\section{CONCLUSIONES}

Como ya se mencionó anteriormente, un hecho que puede presentarse cuando se trabaja con la optimización simultánea de múltiples respuestas, es que en algunas ocasiones pudiera pretenderse llegar a resultados que cuiden más los valores de algunas variables sin importar que se relajen un poco en algunas otras, es decir, no siempre todas las variables consideradas serán igualmente importantes. En este trabajo se utilizó la metodología propuesta por Miró-Quesada et al.,(2004) por ser una de las más completas en el campo de la optimización de múltiples respuestas, ya que considera las correlaciones entre los datos, la incertidumbre en los parámetros de los modelos y la robustez a factores de ruido y además, la variable a optimizar es la probabilidad de conformancia, la cual es fácilmente entendida por los ingenieros a cargo de los procesos, solo que esta metodología fue modificada en los dos aspectos que a continuación se mencionan.

En primer lugar para incorporar las diferentes ponderaciones para las variables de respuesta de acuerdo a su importancia, se sustituyó la probabilidad de conformancia simultánea de todas las variables de respuesta, por la media geométrica ponderada de las probabilidades de conformancia individuales de cada una de las respuestas y en segundo lugar se utilizó el método del gradiente reducido generalizado en la fase de optimización, que permite la búsqueda restringida y que además se encuentra disponible en el paquete Solver de Excel, lo que lo hace bastante accesible para cualquier usuario.

Fase $X$ : En los resultados que se muestran en el ejemplo para ilustrar la metodología con las modificaciones planteadas, se puede ver primeramente que esta funciona adecuadamente, ya que lleva a resultados que son coherentes con la información que se tiene. Por otra parte en lo que se refiere a las diferentes ponderaciones posibles a las variables de respuesta, se puede ver que estas realmente pueden afectar los resultados obtenidos. En la tabla 7 puede apreciarse que los resultados respecto a los valores de $x_{2}$ y $x_{3}$ en $M g p_{1}, M g p_{2}$ y $M g p_{3}$ son similares, sin embargo, también se observa que si se compara $M g p_{2}$ con $M g p_{1}$, el procedimiento por si solo busca condiciones en donde se comporte de mejor manera $y_{1}$ sin afectar demasiado a $y_{2}$, debido a que sus respectivas ponderaciones no son tan diferentes, pero si se compara $M g p_{3}$ con $M g p_{1}$ las modificaciones a favor de $y_{1}$ y en contra de $y_{2}$ se empiezan a hacer más evidentes, esto debido a que las diferencias en las ponderaciones se hacen más grandes a favor de $y_{1}$. Continuando con las comparaciones, al considerar a $\mathrm{Mpg}_{4}$, se puede observar que los valores óptimos propuestos tanto para $x_{2}$ como para $x_{3}$, ahora sí son bastante diferentes a los anteriores, sobre todo en $x_{2}$, esto es debido a que en esta ocasión se le dio una ponderación de 0.2 a $y_{1}$ y de 0.8 a $y_{2}$, por lo que la metodología busca la zona en donde $y_{2}$ tuvo su mejor desempeño castigando a $y_{1}$, lo cual puede apreciarse en las probabilidades y las medias de la tabla 7.

Adicionalmente en la tabla 3 puede observarse que $\mathrm{Mgp}_{4}$ tiene su resultado más alto en $\mathrm{x}_{2}=1.3456$ y $x_{3}=-0.6728$ por lo que el resultado óptimo alcanzado es muy similar a este según la tabla 7 . Respecto a $M g p_{1}$ también tiene su mejor resultado en $x_{2}=1.3456$ y $x_{3}=-0.6728$ de acuerdo con la tabla 3 , sin embargo su resultado óptimo se encontró en $x_{2}=1.6630$ y $x_{3}=-0.7670$, esto fue debido a que $M g p_{1}$ también se comporta de buena manera en $x_{2}=1.682$, de hecho su segundo y tercer resultados más altos se encuentran en esta zona con valores muy cercanos al primero. Es importante señalar también que al resolver el problema presentado como ejemplo en este trabajo, Myers y Montgomery (1995) encuentran como mejor propuesta para solución óptima $\mathrm{x}_{2}=1.682$ y $\mathrm{x}_{3}=-0.5955$, la cual es similar a las encontradas utilizando $M g p_{1}$ y $M g p_{2}$ y es casi idéntica a la obtenida con $\mathrm{Mgp}_{3}$.

Con lo anterior entonces se puede concluir que la metodología funciona adecuadamente con las modificaciones propuestas y que dar ponderaciones diferentes a las variables de respuesta, sí puede modificar los resultados óptimos alcanzados, buscando configuraciones del proceso con mejores comportamientos en las variables con los mayores pesos. Esto último hace posible analizar casos en los que no todas las respuestas son igualmente importantes, con lo que la metodología se vuelve más flexible y atractiva en muchos casos prácticos, ya que en su diseño original no permitía incorporar estas consideraciones. Una ventaja adicional es que con la 
modificación propuesta acerca del uso de ponderaciones, el problema se puede resolver utilizando diferentes combinaciones de pesos, lo que proporcionaría a los ingenieros o a los investigadores diferentes alternativas óptimas de operación del proceso para escoger, tomando en consideración algunos otros aspectos prácticos del propio proceso, lo que puede facilitar y enriquecer la toma de decisiones.

\section{REFERENCIAS}

Ames, A. E., y otros cuatro autores, Quality Loss Function for optimization across multiple response surface, Journal of Quality Technology, 29(3), 339-346, (1997).

Ch'ng, C. K., Quah S. H. y Low H. C., Index C*pm in multiple response optimization, Quality Engineering, 17(1), 165-171, (2005).

Chiao, C. H. y Hamada, M., Analyzing experiments with correlated multiple responses, Journal of Quality Technology, 33(4), 451-465, (2001).

Del Castillo, E., Montgomery, D. C. y McCarville, D. R., Modified desirability functions for multiple response optimization, Journal of quality technology, 28(3), 337-345, (1996).

Derringer, G. y Suich, R., Simultaneous optimization of several response variables, Journal of Quality Technology, 12, 214-219, (1980).

Ko, Y. H., Kim, K. J. y Jun, C. H., A new loss function-based method for multiresponse optimization, Journal of Quality Technology, 37(1), 50-59, (2005).

Limón, J., y otros tres autores, Evaluación de la robustez del sistema Mahalanobis-Taguchi a diferentes arreglos factoriales, Información Tecnológica, 22(4), 85-96, (2011).

Miró-Quesada, G., Del Castillo, E. y Peterson, J. J., A Bayesian approach for multiple response surface optimization in the presence of noise variables, Journal of Applied Statistics, 3(3), 251-270, (2004).

Myers, R. H. y Montgomery, D. C., Response Surface Methodology Process and Product Optimization Using Designed Experiments, $1^{\text {a }}$ edición, 244-265, Wiley Series in Probability and Statistics, New York, Estados Unidos de America, (1995).

Noorossana, R. y Ardakani, M. K., A weighted metric method to optimize multi-response robust problems, Journal of Industrial Engineering International, 5(8), 10-19, (2010).

Peterson, J. J., A posterior predictive approach to multiple response surface optimization, Journal of Quality Technology, 36(2), 139-153, (2004).

Plante, R. D., Process capability: a criterion for optimizing multiple response product and process design, IIE Transactions, 33, 497-509, (2001). 
